\title{
Analysis of the Effect of a Gyrotropic Anisotropy on the Phase Constant and Characteristic Impedance of a Shielded Microstrip Line
}

\author{
Djamel Sayad ${ }^{1}$, Chemseddine Zebiri², Samiha Daoudi ${ }^{3}$, and Fatiha Benabdelaziz \\ ${ }^{1}$ Département de Génie Electrique, Université 20 Août 1955-Skikda, Skikda, Algeria \\ ${ }^{2}$ Département d'Electronique, Université Ferhat Abbas, Sétif 1, Sétif, Algeria \\ ${ }^{3}$ Département d'Electronique, Université Mentouri, Constantine 1, Constantine, Algeria \\ ${ }^{1}$ Corresponding author, E-mail: czebiri@univ-setif.dz; d.sayad@univ-skikda.dz
}

\begin{abstract}
In this work, we present an analytical modeling of a highly complex medium-based shielded microstrip line. The study aims at a numerical evaluation of the characteristic impedance and the dispersion characteristics of the dominant hybrid mode in the microstrip line printed on an anisotropic medium. The newly considered complex anisotropy has a full $3 \times 3$ tensor form of permittivity and permeability. The study is based on the derivation of the Green's functions of the general complex-medium-based structure in the Fourier domain. The spectral Method of Moments (MoM) and the Galerkin's procedure are combined to solve the resulting homogeneous system of equations. The effect of the gyrotropic anisotropy on the phase constant and the characteristic impedance is particularly investigated. Original and interesting numerical results are obtained and discussed. Our results are found to be in good agreement with available isotropic case data reported in literature.
\end{abstract}

\section{Introduction}

Because of the recent tendency towards miniaturization and integration, microwave circuitry is currently fabricated using integrated transmission lines, because of their planar layout, rather than bulky waveguides. This feature is highly attractive for higher frequency band and higher packing density device technologies $[1,2]$.

Due to their many potential applications in a large number of practical problems in electromagnetic, optic and acoustic domains, anisotropic media have attracted much interest and support from researchers and industrials as powerful instruments with a real future in microwave and optic engineering [3,4]. Recently, various anisotropic medium structures are intensively employed in optical systems, optoelectronics, antenna [5], radar absorbers and microwave devices [6][7]. Their anisotropic nature is an attractive characteristic for advanced electronic application devices based on the nonreciprocity property of complex media $[8,9]$. In this class of complex materials, we find, for instance, ferrite, chiral, and metamaterial which all seem to include a great potential for promising applications in microwave, infra-red and optical domains [10-13]. This anisotropy results in additional degrees of freedom for the design of electromagnetic devices and leads to new fields of applications due to the introduction of new synthetic electronic materials $[14,15]$.

A deep investigation of the material response to electromagnetic waves may even allow the design and fabrication of materials with properties not found in nature. Microwave bi-anisotropic materials can be realized as microstructures comprising small metal helices distributed in isotropic matrices. When the helices are arranged in a certain order, we obtain, for example, chiral media. One of them is the so-called omega composite that consists of embedded small $\Omega$-shaped inclusions in an isotropic dielectric matrix [16]. The origin of the anisotropy is either the nonspherical shape of the metallic inclusions in the 3D systems, or the distribution of the inclusions (even if spherical) on a substrate in the $2 \mathrm{D}$ configuration. In both cases, it leads to an anisotropic effective medium [17]. Metamaterials are composite structures made of metallic inclusions such as rods and split rings resonators properly arranged in space in order to achieve negative values of the permittivity and the permeability. Ferrites have a crystal structure sintered by means of various metal oxides. In the absence of an applied static field, demagnetized ferrites are isotropic materials. In the presence of a static magnetic field, ferrites exhibit a nonreciprocal anisotropic behavior [18].

To extract the effective constitutive parameters of anisotropic materials, various techniques such as stepwise method, S-parameters method, resonator method, coaxial probe method, free-space characterization method, rectangular waveguide measurements and recursive algorithms have been employed [19] [20] [21].

It is crucial to predict the electromagnetic behavior of a general anisotropic medium for their optimal use in the design of transmission lines. Therefore, it is basically essential to be able to evaluate the transmission line characteristic impedance in all the frequency band of operation. Reported results of the propagation characterization in anisotropic substrate transmission lines, show that the electromagnetic parameters of the substrate 
present dominant effects on the wave propagation characteristics and they are quite different according to the type of used substrates [22-24].

Exact solutions of electromagnetic radiation and propagation problems have become an increasingly important area of research. There have been many works reported in literature using spectral domain technique in analyzing microstrip lines [25-22]. Solving electromagnetic field problems, analysis and designs need numerical computational methods to be employed. Various methods and techniques maybe used to investigate and optimize mono and multilayered microstrip structures [23,29,30].

To predict accurately the electromagnetic behavior of transmission lines over a given frequency band, the Moment Method is used in the spectral domain to obtain a solution. This technique is commonly used for planar structures such as microstrip lines and antennas with perfect conductors, and it is first applied in microwave domain by Harrington in 1968 [31-34].

\section{Analytical formulation}

The general microstrip line geometry and the appropriate coordinate system with the $z$-axis as direction of propagation are shown in Fig. 1. The metal involved in the structure is assumed to be perfect conductor.

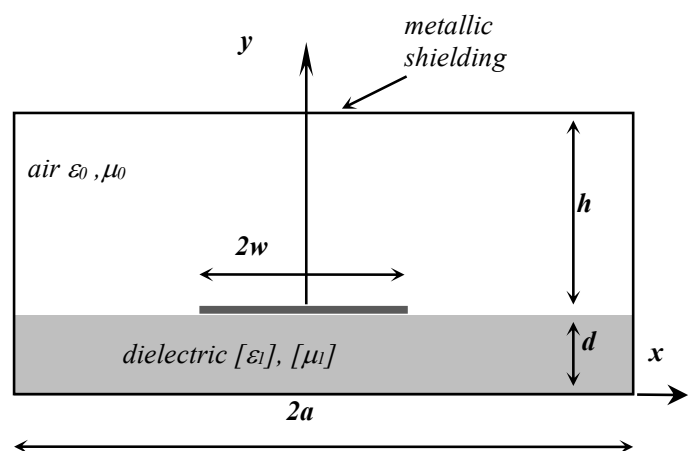

Figure 1: Configuration of a shielded microstrip.

In this study, we consider a most general complex anisotropic medium (region 1) characterized by full $3 \times 3$ permittivity and permeability tensors. For this medium, the following conditions on the permittivity and permeability constants are considered $\bar{\varepsilon}=\bar{\varepsilon}^{T}, \quad \bar{\mu}=\bar{\mu}^{T}$ and additional conditions are necessary to ensure the decoupling of the TE and TM modes independently of the direction of propagation in the medium [35]:

$\varepsilon_{x y}+\varepsilon_{y x}=0, \quad \varepsilon_{x z}+\varepsilon_{z x}=0, \quad \varepsilon_{y z}+\varepsilon_{z y}=0 \quad$ and $\quad \bar{\mu}=\tau \bar{\varepsilon}^{T}$, which gives:

$$
\bar{\varepsilon}=\varepsilon_{0}\left[\begin{array}{ccc}
\varepsilon_{x x} & \varepsilon_{x y} & \varepsilon_{x z} \\
-\varepsilon_{x y} & \varepsilon_{y y} & \varepsilon_{y z} \\
-\varepsilon_{x z} & -\varepsilon_{y z} & \varepsilon_{z z}
\end{array}\right],
$$

and

$$
\bar{\mu}=\mu_{0} \tau\left[\begin{array}{ccc}
\varepsilon_{x x} & -\varepsilon_{x y} & -\varepsilon_{x z} \\
\varepsilon_{x y} & \varepsilon_{y y} & -\varepsilon_{y z} \\
\varepsilon_{x z} & \varepsilon_{y z} & \varepsilon_{z z}
\end{array}\right],
$$

Assuming the temporal dependence $e^{j \omega t}$ of the fields and considering Maxwell's equations in the Fourier domain with $\partial / \partial z \equiv-j \beta$ and $\partial / \partial x \equiv-j \alpha$ assumptions, we derive the transverse electromagnetic field components as functions of the longitudinal electric and magnetic components $\widetilde{E}_{z 1}$ and $\widetilde{H}_{z 1}$ in region 1 .

$$
\begin{aligned}
\widetilde{E}_{x 1}= & \frac{1}{\delta_{1}}\left(\alpha_{n} \beta+\kappa_{0}^{2} \tau\left(\varepsilon_{y y} \varepsilon_{x z}-\varepsilon_{x y} \varepsilon_{y z}\right)\right) \widetilde{E}_{z 1} \\
& +\frac{\omega \mu_{0} \tau}{\delta_{1}}\left(\varepsilon_{x y} \alpha_{n}-\varepsilon_{y z} \beta+\varepsilon_{y y} j \frac{\partial}{\partial y}\right) \widetilde{H}_{z 1} \\
\widetilde{E}_{y 1}= & \frac{1}{\delta_{1}}\left(\kappa_{0}^{2} \tau\left(\varepsilon_{x x} \varepsilon_{y z}+\varepsilon_{x y} \varepsilon_{x z}\right)+j \beta \frac{\partial}{\partial y}\right) \widetilde{E}_{z 1} \\
& +\frac{\omega \mu_{0} \tau}{\delta_{1}}\left(\varepsilon_{x z} \beta-\varepsilon_{x x} \alpha_{n}+\varepsilon_{x y} j \frac{\partial}{\partial y}\right) \widetilde{H}_{z 1} \\
\widetilde{H}_{x 1}= & \frac{\omega \varepsilon_{0}}{\delta_{1}}\left(\varepsilon_{x y} \alpha_{n}-\varepsilon_{y z} \beta-\varepsilon_{y y} j \frac{\partial}{\partial y}\right) \widetilde{E}_{z 1} \\
& +\frac{1}{\delta_{1}}\left(\alpha_{n} \beta-\kappa_{0}^{2} \tau\left(\varepsilon_{x y} \varepsilon_{y z}+\varepsilon_{y y} \varepsilon_{x z}\right)\right) \widetilde{H}_{z 1},(2 c) \\
\widetilde{H}_{y 1}= & \frac{\omega \varepsilon_{0}}{\delta_{1}}\left(\varepsilon_{x x} \alpha_{n}+\varepsilon_{x z} \beta+\varepsilon_{x y} j \frac{\partial}{\partial y}\right) \widetilde{E}_{z 1} \\
& +\frac{1}{\delta_{1}}\left(\kappa_{0}^{2} \tau\left(\varepsilon_{x y} \varepsilon_{x z}-\varepsilon_{x x} \varepsilon_{y z}\right)+j \beta \frac{\partial}{\partial y}\right) \widetilde{H}_{z 1}
\end{aligned}
$$

With

$$
\delta_{1}=\beta^{2}-\kappa_{0}^{2} \tau\left(\varepsilon_{x x} \varepsilon_{y y}+\varepsilon_{x y}^{2}\right),
$$

which results in the following complex wave equation for the components $\widetilde{E}_{z 1}$ and $\widetilde{H}_{z 1}$ given in the Fourier domain:

$$
\frac{\partial^{2} \widetilde{\Psi}_{1}}{\partial y^{2}}-\gamma_{1}^{2} \widetilde{\Psi}_{1}=0
$$

where $\widetilde{\Psi}_{1}$ stands for longitudinal components $\widetilde{E}_{z 1}$ or $\widetilde{H}_{z 1}$, and the dispersion relation for such an unbounded complex medium is found to be

$$
\gamma_{1}^{2}=\frac{\varepsilon_{x x}}{\varepsilon_{y y}} \alpha_{n}^{2}+\frac{\varepsilon_{z z}}{\varepsilon_{y y}} \beta^{2}-\kappa_{0}^{2} \tau\left(\varepsilon_{x x} \varepsilon_{z z}+\frac{\varepsilon_{z z}}{\varepsilon_{y y}} \varepsilon_{x y}^{2}+\varepsilon_{x z}^{2}+\frac{\varepsilon_{x x}}{\varepsilon_{y y}} \varepsilon_{y z}^{2}\right)(4)
$$

Due to the perfect side walls of microstrip structure, electromagnetic fields are only defined for $x$ from $-a$ to $a$ (Fig. 1). $\alpha_{n}$ is the discrete Fourier transform variable, with 
values for even modes: $\alpha_{n}=(n-0.5) \pi / a$ and for odd modes $\alpha_{n}=n \pi / a$, defined within the microstrip line [26,36,37].

\section{Method of solution}

Eq. (3) admits a general solution, in the bounded complex medium region, of the form

$$
\widetilde{\Psi}_{1}=A \sinh \left(\gamma_{1} y\right)+B \cosh \left(\gamma_{1} y\right),
$$

where $A$ and $B$ are complex constants.

To determine the complex constants appearing in the expressions of the electromagnetic field components, the following boundary conditions are used.

at $y=0$,

$$
\widetilde{E}_{z 1}=\widetilde{E}_{x 1}=0
$$

and at $\mathrm{y}=\mathrm{d}$,

$$
\begin{aligned}
& \widetilde{E}_{z 2}-\widetilde{E}_{z 1}=0, \\
& \widetilde{E}_{x 2}-\widetilde{E}_{x 1}=0, \\
& \widetilde{H}_{x 2}-\widetilde{H}_{x 1}=-\widetilde{J}_{z}, \\
& \widetilde{H}_{z 2}-\widetilde{H}_{z 1}=\widetilde{J}_{x},
\end{aligned}
$$

where $\tilde{J}_{x}$ and $\tilde{J}_{z}$ are the Fourier transforms of the transverse current density $J_{x}(x)$ and the longitudinal current density $J_{z}(x)$, respectively. These basis functions are chosen such that they are nonzero only on the strip $|x|<w$ at $y=d$.

\subsection{Derivation of Green's functions}

Extensive algebraic manipulations of the resulting mathematical equations lead to the expressions of the electric field evaluated at the interface between the two media with respect to the current densities $\widetilde{J}_{x}$ and $\widetilde{J}_{z}$. Green's tensor elements are determined according to the following system of equations $[31,36]$.

$$
\begin{aligned}
& \widetilde{E}_{z}\left(\alpha_{n}, d, \beta\right)=\widetilde{G}_{11}\left(\alpha_{n}, d, \beta\right) \widetilde{J}_{x}+\widetilde{G}_{12}\left(\alpha_{n}, d, \beta\right) \widetilde{J}_{z}(7 \mathrm{a}) \\
& \widetilde{E}_{x}\left(\alpha_{n}, d, \beta\right)=\widetilde{G}_{21}\left(\alpha_{n}, d, \beta\right) \widetilde{J}_{x}+\widetilde{G}_{22}\left(\alpha_{n}, d, \beta\right) \widetilde{J}_{z}(7 \mathrm{~b})
\end{aligned}
$$

\subsection{Dispersion characteristics}

For a given frequency the propagation constant can be obtained by setting the determinant of deduced matrix from Equation (7) equal to zero (to have non-trivial solutions) and searching for the root of the resulting equation [28].

\subsection{Evaluation of the characteristic impedance}

Because of the non-TEM nature of the propagation mode, there are more than one definition for the characteristic impedance. We used herein the power current definition to calculate the characteristic impedance [38].

$$
Z_{c}=\frac{2 P}{I_{0}^{2}}
$$

where $I_{0}$ is the effective current flow along the $z$ axis on the strip and the power is given by the flux of the Poynting vector through the microstrip line cross section

$$
P=\frac{1}{2} \operatorname{Re} \int_{0}^{h} \int_{-a}^{a} \vec{E} \times \vec{H}^{*} \cdot \vec{u}_{z} \cdot d x \cdot d y,
$$

\section{Results and discussion}

To validate the developed analytical formulations, we have firstly calculated the dispersion characteristics $\beta$ of an isotropic-medium shielded microstrip transmission line case, evaluated for the following structure parameters: $d=0.635 \mathrm{~mm}, \quad h=4 d, \quad a=10 d, \quad w=d / 2$, and by setting $\left(\varepsilon_{x x}=\varepsilon_{y y}=\varepsilon_{z z}=\varepsilon_{r l}=10, \quad \varepsilon_{x y}=\varepsilon_{x z}=\varepsilon_{y z}=0, \quad \tau=1 / \varepsilon_{r l}\right), \quad$ for a frequency range up to $15 \mathrm{GHz}$. Fig. 2 shows good agreements with published results [26].

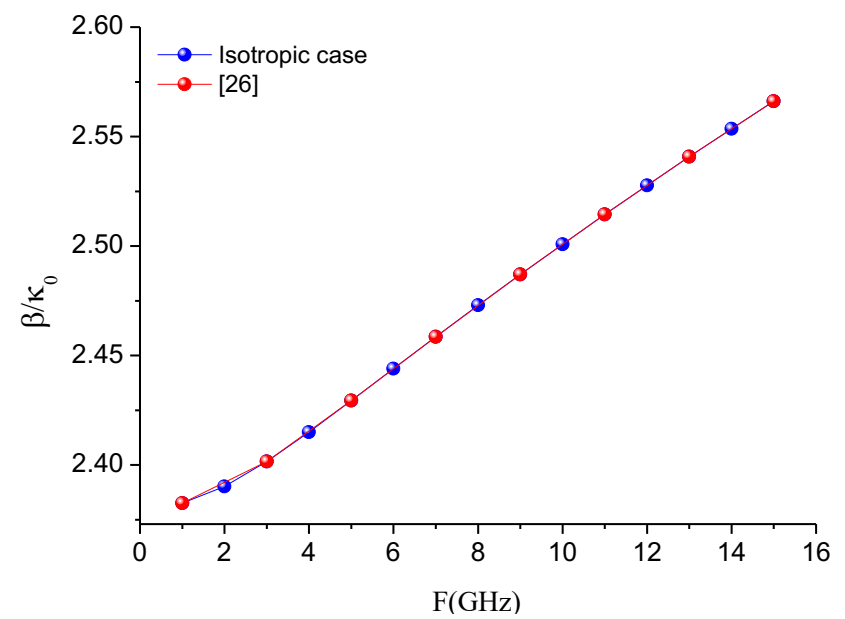

Figure 2: Dispersion characteristics of the dominant mode compared with literature.

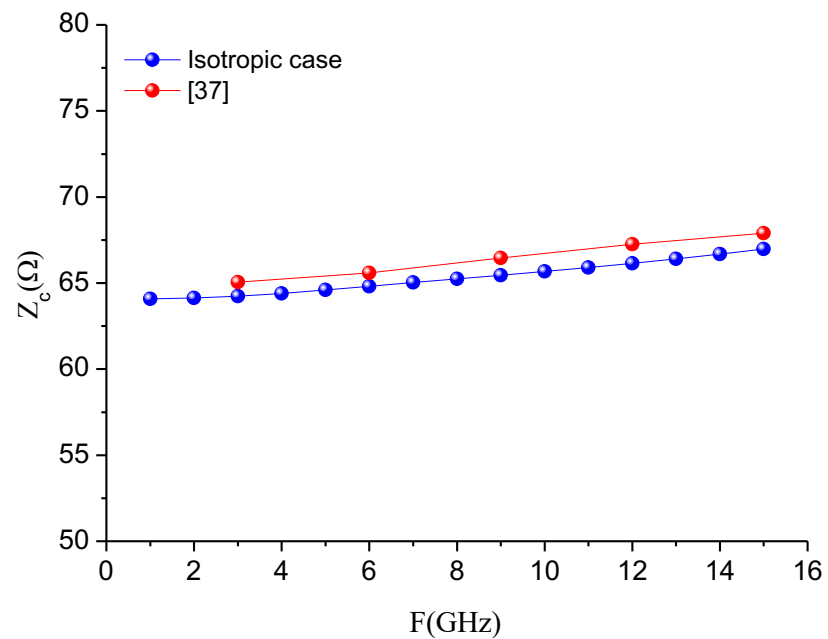

Figure 3: Calculated characteristic impedance compared with literature.

A comparison representation of the characteristic impedance for the same configuration parameters used 
above is given in Fig. 3. The representation shows good agreements with available experimental data reported in literature [37].

\subsection{Effect of the anisotropy on the phase constant and the characteristic impedance}

Initially, we treat the case of uniaxial anisotropy by examining the effect of the diagonal elements of the tensors of permittivity and permeability on the phase constant $\beta$ and the characteristic impedance $Z c$ with respect to frequency. The other gyrotropic elements $\left(\varepsilon_{x y}, \varepsilon_{x z}, \varepsilon_{y z}\right)$ are taken to be null and $\tau=1 / \varepsilon_{r}$. The obtained results are presented by Fig. 4 , and are compared with the isotropic case $\left(\varepsilon_{r l}=10\right)$, for the following dimensional parameters $(d=0.635 \mathrm{~mm}, h=4 d$, $a=10 d, w=d / 2$ ).

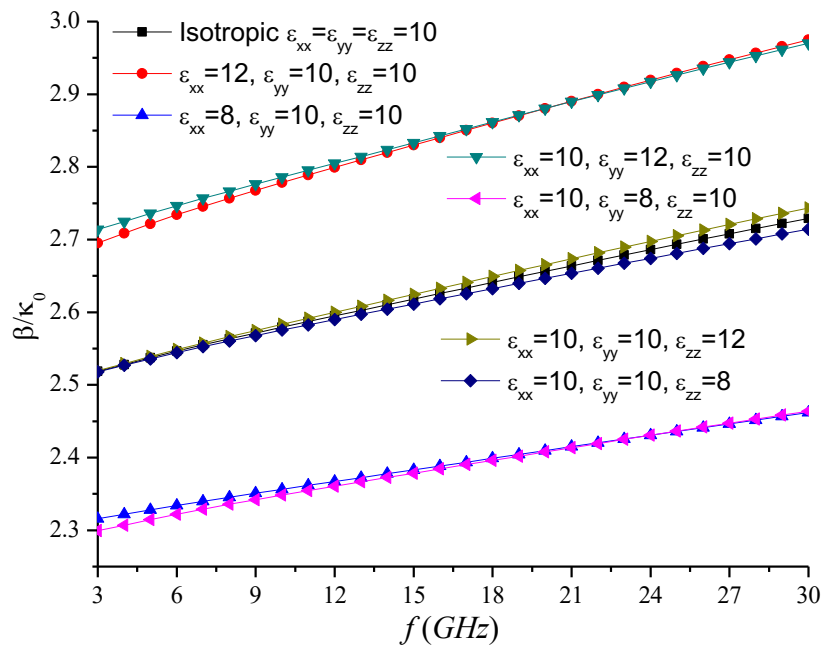

Figure 4.a: Effect of uniaxial anisotropy on the normalized phase constant

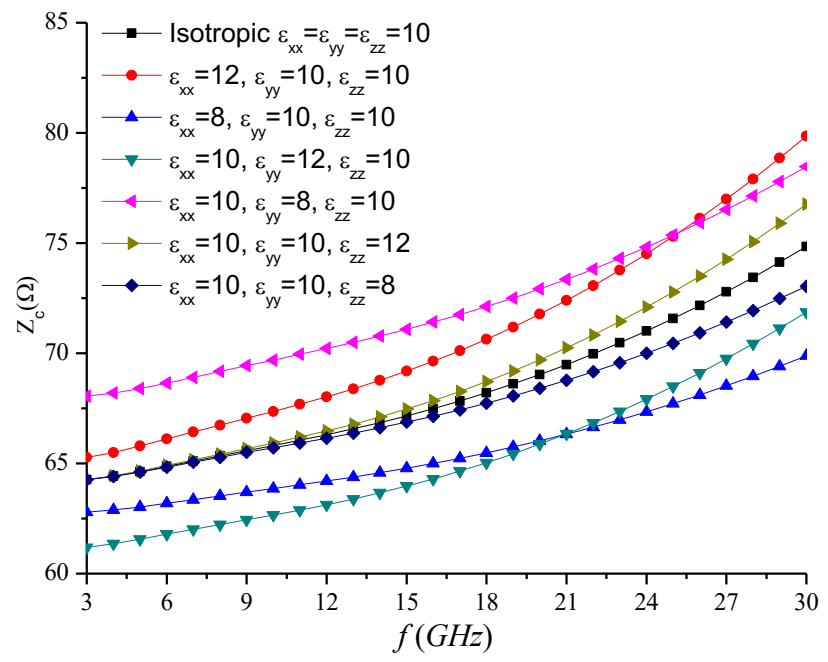

Figure 4.b: Effect of uniaxial anisotropy on the characteristic impedance

According to these results, we notice that $\varepsilon_{z z}$ is the least influential element on $\beta$ and $Z_{c}$ and in particular in the range of lower frequencies. The elements $\varepsilon_{x x}$ and $\varepsilon_{y y}$ have almost the same influence on $\beta$, and the variation of this latter is directly proportional to the variation of these two elements.

For the characteristic impedance, it is directly proportional to $\varepsilon_{x x}$ and inversely proportional to $\varepsilon_{y y}$. It should be noted that the characteristic impedance presents the weakest variation with respect to frequency in the case of negative anisotropy of the element $\varepsilon_{x x}$, (i.e. $\varepsilon_{x x}=0.8 \varepsilon_{r l}<\varepsilon_{r l}$ ), and the most important variation in the case of positive anisotropy of the same element (i.e. $\varepsilon_{x x}=1.2 \varepsilon_{r l}>\varepsilon_{r l}$ ).

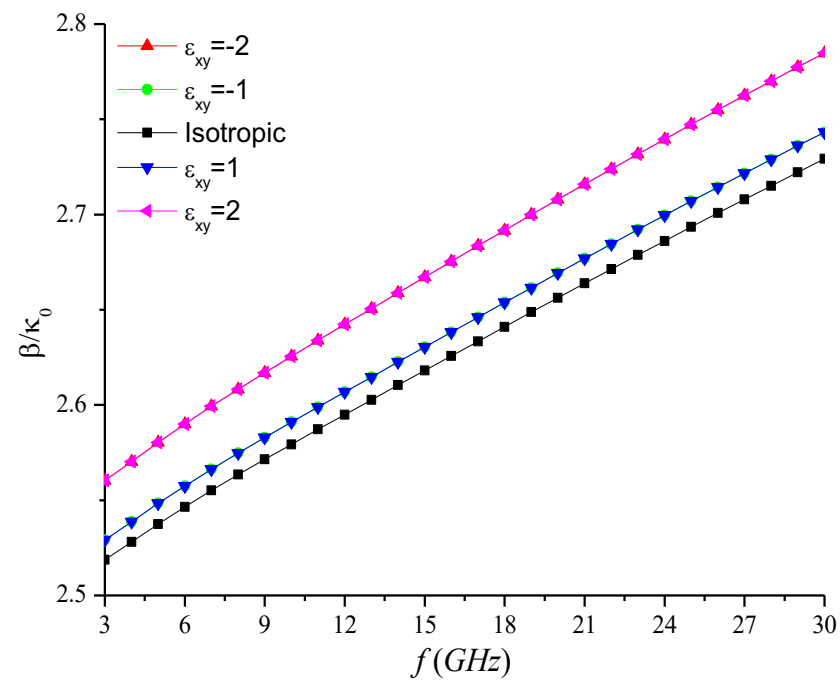

Figure 5.a: Effect of real valued $\varepsilon_{x y}$ on the normalized phase constant

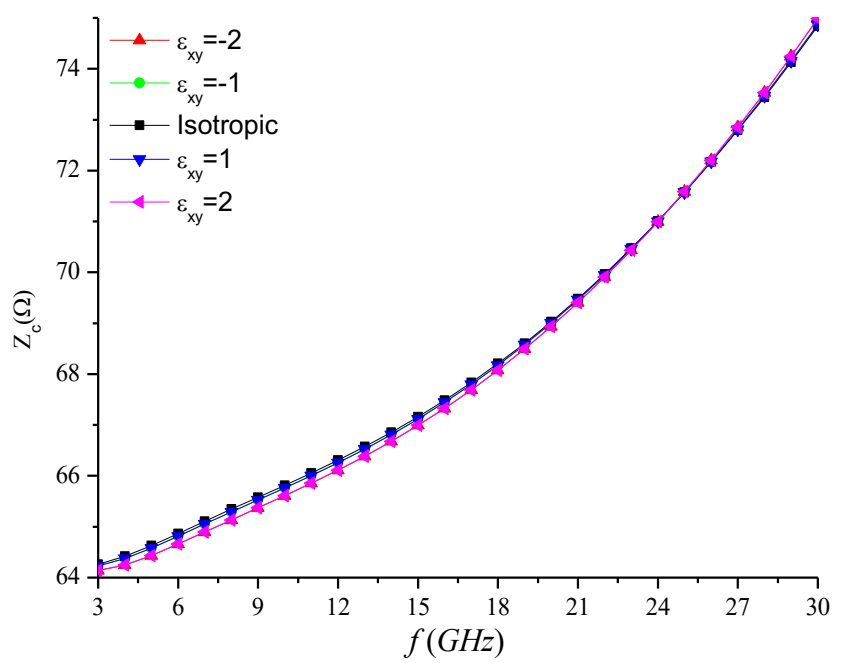

Figure 5.b: Effect of real valued $\varepsilon_{x y}$ on the characteristic impedance

Now, we examine the case of gyrotropic anisotropy by varying the higher and lower elements of permittivity and permeability matrix $\varepsilon_{x y}, \varepsilon_{x z}$ and $\varepsilon_{y z}$ : non diagonal elements which can take positive, negative, real or imaginary values. In this case the diagonal elements are taken equal to the isotropic case permittivity $\left(\varepsilon_{x x}=\varepsilon_{y y}=\varepsilon_{z z}=\varepsilon_{r l}=10\right)$. The obtained results for each element are shown on Figs. 5-10, compared to the isotropic case. Figs. 5 and 6 present the $\varepsilon_{x y}$ 
effect. This latter presents an important effect on $\beta$. The increase in absolute value of real valued $\varepsilon_{x y}$ leads to an increase in the phase constant and with no effect on $Z_{c}$ (Fig. $5(\mathrm{~b})$ ).

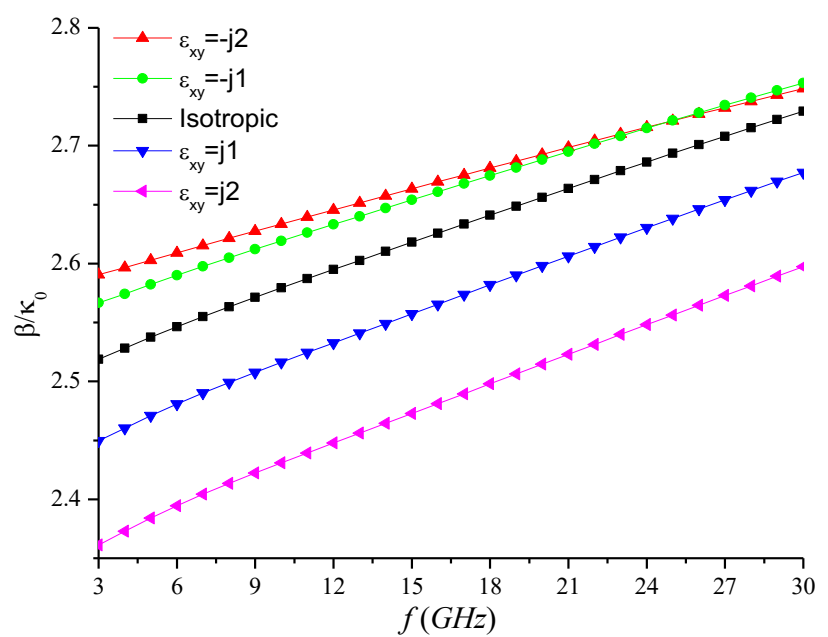

Figure 6.a: Effect of imaginary valued $\varepsilon_{x y}$ on the normalized phase constant

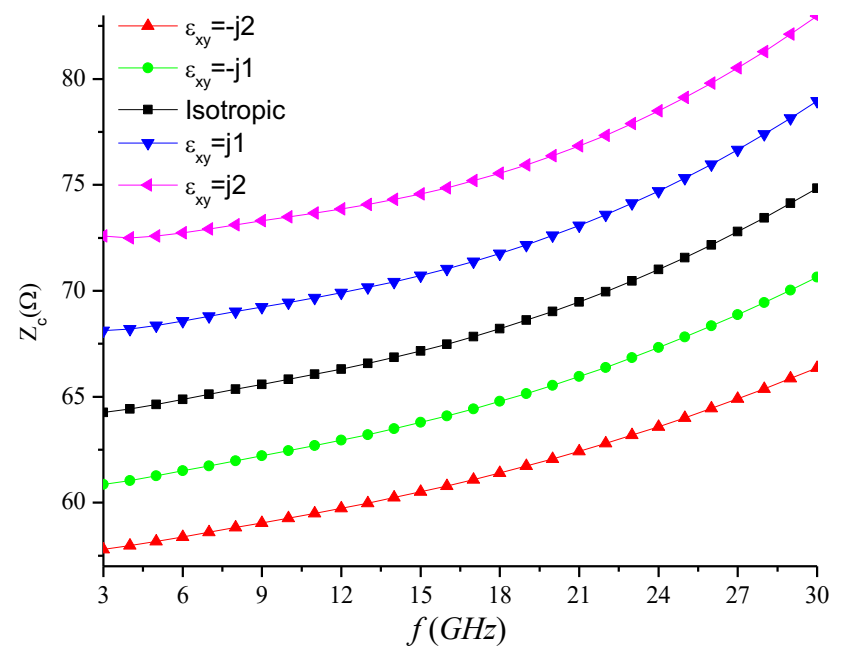

Figure 6.b: Effect of imaginary valued $\varepsilon_{x y}$ on the characteristic impedance

For imaginary values, $\varepsilon_{x y}$ presents an important effect on $\beta$ and $Z_{c}$. The positive values have more effect on $\beta$ and practically the same effect on $Z c$ as negative values by increasing $\beta$ and decreasing $Z c$ (Fig. 6(a) and (b)).

The gyrotropic anisotropy associated with the element $\varepsilon_{y z}$ behaves differently compared to $\varepsilon_{x y}$ towards $\beta$ and $Z_{c}$. For real values of $\varepsilon_{y z}$, the effect is negligible either on $\beta$ or $Z_{c}$ (Fig. 7).

Whereas for imaginary values (Fig. 8), this element has less important effect than $\varepsilon_{x y}$ on $\beta$, and an effect on $Z_{c}$ which increases more and more with frequency contrary to the element $\varepsilon_{x y}$ (Fig. 6).

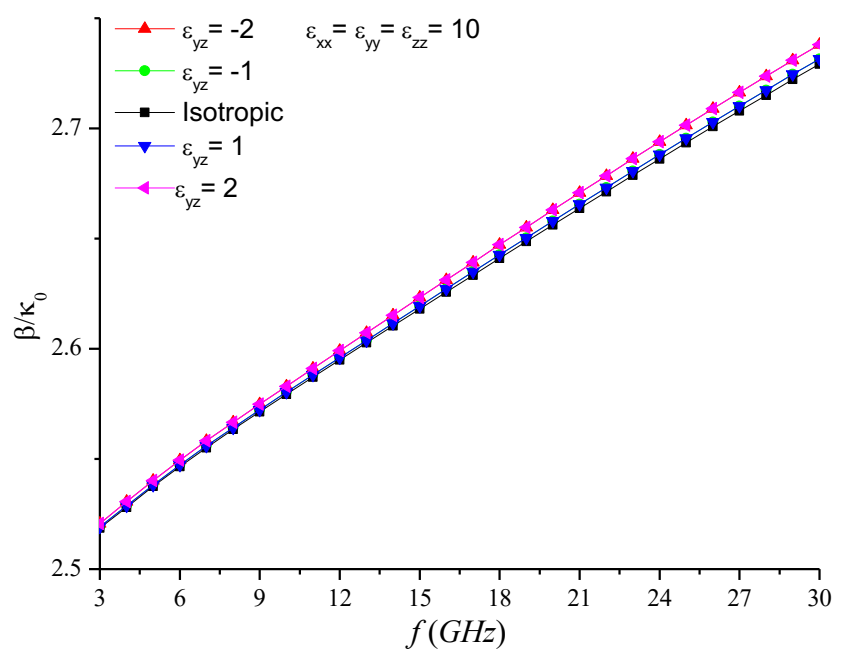

Figure 7.a: Effect of real valued $\varepsilon_{y z}$ on the normalized phase constant

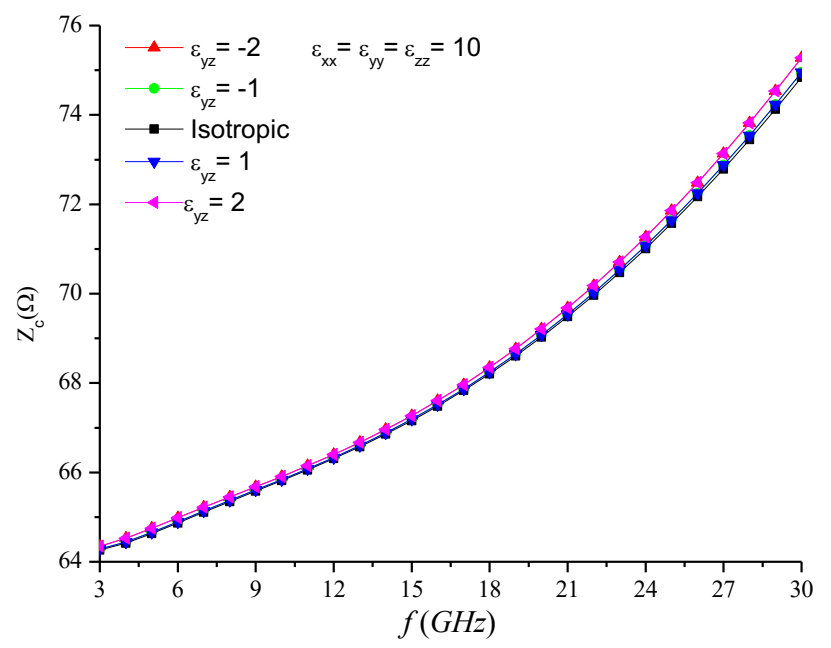

Figure 7.b: Effect of real valued $\varepsilon_{y z}$ on the characteristic impedance

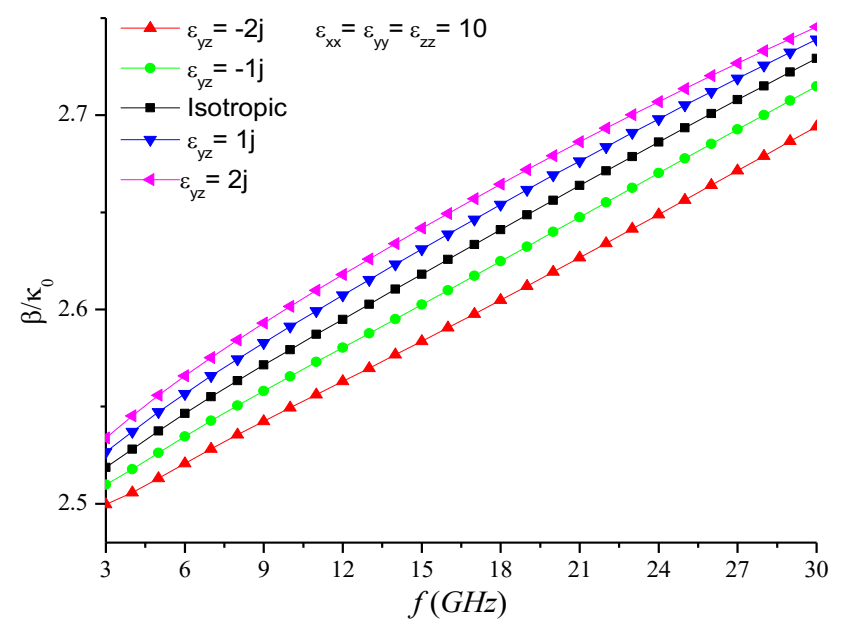

Figure 8.a: Effect of imaginary valued $\varepsilon_{y z}$ the normalized phase constant 


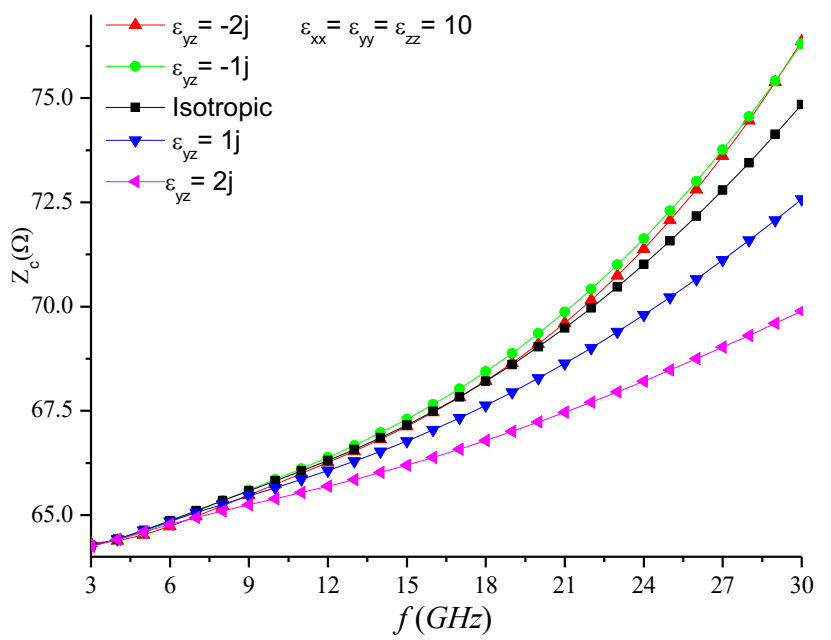

Figure 8.b: Effect of imaginary valued $\varepsilon_{y z}$ on the characteristic impedance

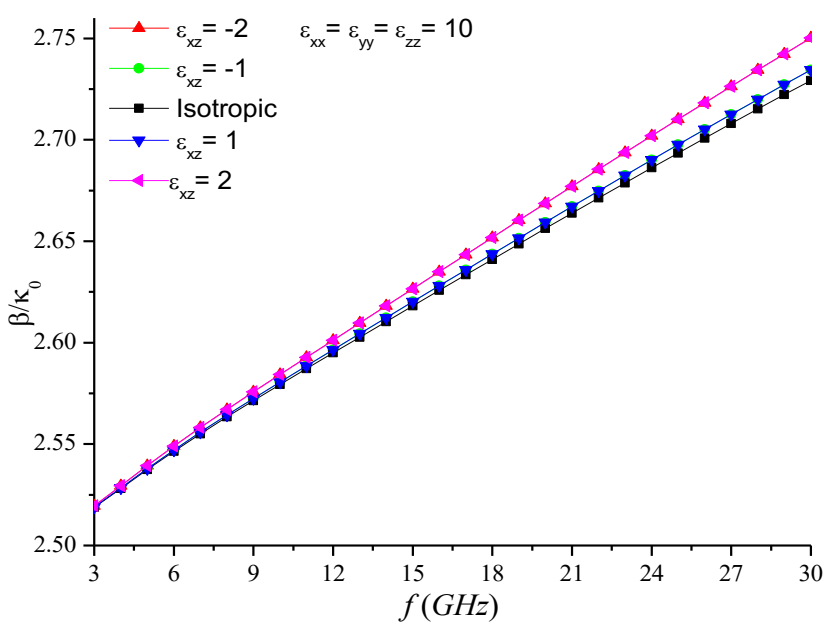

Figure 9.a: Effect of real valued $\varepsilon_{x z}$ the normalized phase constant

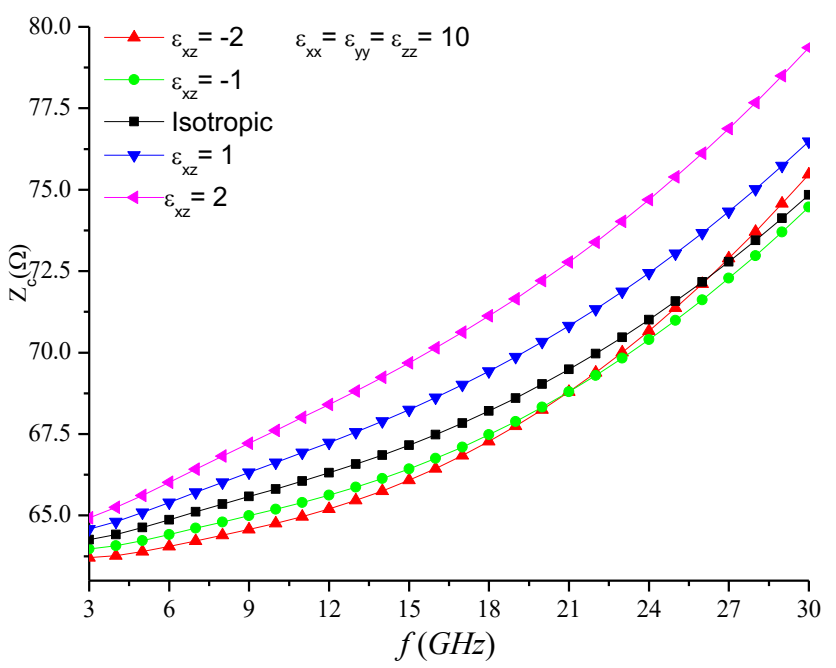

Figure 9.b: Effect real valued $\varepsilon_{x z}$ on the characteristic impedance
The effect of the real values of the element $\varepsilon_{x z}$ on $\beta$ (Fig. $9(a))$ is almost the same as that of imaginary values with opposite sign (Fig. 10(a)). For the effect of this element on $Z_{c}$, it is completely different compared to the other elements: for $\varepsilon_{x z}$ real (Fig. 9), the variations of $\beta$ and $Z_{c}$ are directly proportional, which is the reverse for imaginary values. Moreover, the variation of $Z_{c}$ is more significant for real valued $\varepsilon_{x z}$ and is directly proportional (Fig. 9(b)).

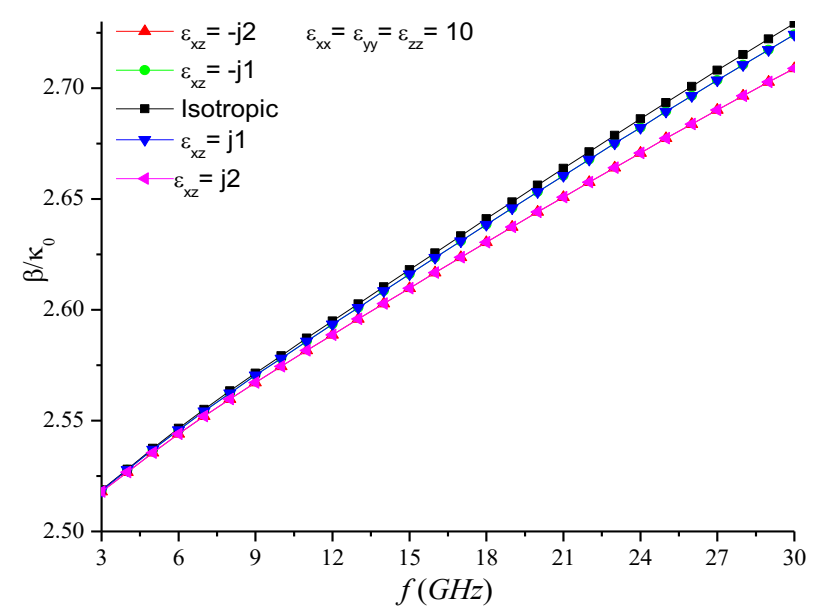

Figure 10.a: Effect of imaginary valued $\varepsilon_{x z}$ the normalized phase constant

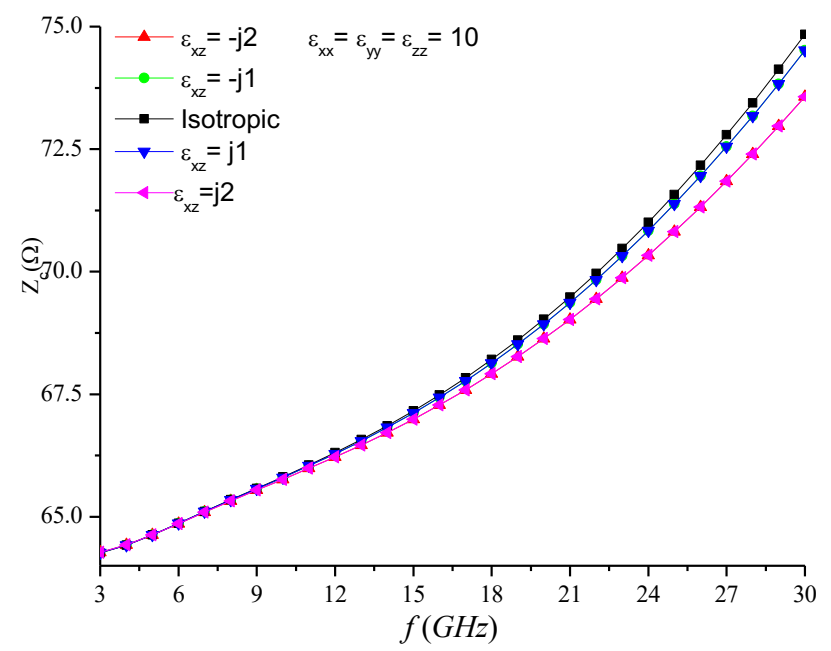

Figure 10.b: Effect of imaginary valued $\varepsilon_{x z}$ on the characteristic impedance

Fig. 11, gives a more favorable selection of a combination of gyrotropic anisotropy elements for the characteristic impedance. It should be noted that among these combinations, those with imaginary values are most influential on the characteristic impedance. Moreover, they present minor variations of the characteristic impedance with respect to frequency. This variation is about $3.95 \Omega$ for a $3-30 \mathrm{GHz}$ frequency range for the combination $\varepsilon_{x y}=2 j$, $\varepsilon_{x z}=2 j, \quad \varepsilon_{y z}=2 j$, and about $3.89 \Omega$ for the combination $\varepsilon_{x y}=-$ $2 j, \varepsilon_{x z}=-2 j, \varepsilon_{y z}=2 j$. 


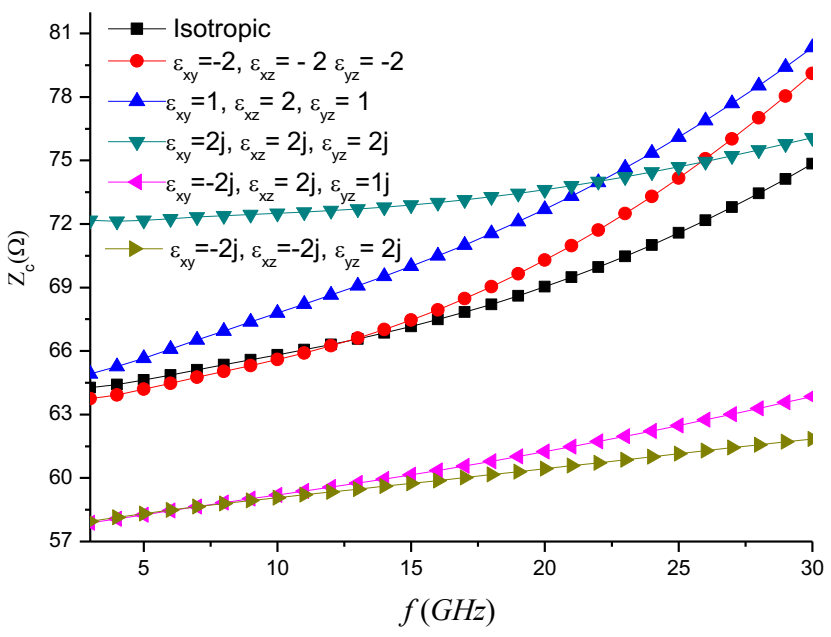

Figure 11: Effect of the gyrotropic anisotropy on the characteristic impedance

Though in this study we considered the effect of each parameter individually, results showed that the combined effects are not mutually compensated in a predicted way, i.e. the coexisting elements will mutually interact and affect the propagation and the characteristic impedance. The individual element effect is not quite absolute or independent, this is due to the above considered conditions that are necessary to ensure the decoupling of the TE and TM modes especially Eq. (1b).

\section{Conclusions}

In this work, a complex and rigorous characterization of a planar transmission line printed on an anisotropic substrate is presented employing the method of moments formulated in the spectral domain. The anisotropic effect of the permittivity elements on the phase constant and the characteristic impedance is analyzed. Original results were carried out, commented on and compared with isotropic case available in literature, good agreement is reached with experimental results of the isotropic characteristic impedance, which validates our theoretical calculations as well as the numerical method of resolution adequately adopted to our complex medium case.

It is worth noting that the effect of the gyrotropic anisotropy permittivity is completely different from the uniaxial anisotropy and the behavior of each element of the permittivity tensor particularly differs from the other elements. This has helped to achieve an optimal combination of the anisotropic elements to reach an optimal variation of the characteristic impedance on a wide frequency range.

These results are the outcome of a full wave technique study which is likely to give exact solutions and a predictive insight of the behavior of anisotropic media.

\section{References}

[1] U. Zaman, V. Vassilev, H. Zirath and N. Rorsman, "Novel Low-Loss Millimeter-Wave Transition From Waveguide-to-
Microstrip Line Suitable for MMIC Integration and Packaging," IEEE Microwave and Wireless Components Letters, Vol., No. 99, pp. 1-3, 2017.

[2] D. Kim, J. M. Woo, H. W. Kim, et al. Quad-band THz frequency selective surface based on a split ring resonator loaded with multiple slots. Journal of Electromagnetic Waves and Applications. Vol. 29, pp. 2472-2478, 2015.

[3] S. Barzegar-Parizi, and B. Rejaei, "Calculation of Effective Parameters of High Permittivity Integrated Artificial Dielectrics," IET Microwaves, Antennas \& Propagation, Vol. 9, No. 12, pp. 1287-1296, 2015.

[4] M. Norooziarab, S. Bulja, R. Cahill, R. Kopf and A. Tate, "Complex Dielectric Permittivity Extraction Based on Multilayer Thin Film Microstrip Lines," IET Microwaves, Antennas \& Propagation, Vol. 11, No. 7, pp. 955-960, 2017.

[5] S. Bedra, S. Benkouda, M. Amir, \& T. Fortaki. Resonant frequency of tunable microstrip ring antenna printed on isotropic or uniaxially anisotropic substrate. Advanced Electromagnetics, Vol. 2(2), 6-9, 2013.

[6] A. Kumar, R. Patel \& M. V. Kartikeyan. Investigation on Microstrip Filters with CSRR Defected Ground Structure. Advanced Electromagnetics, Vol. 5(2), pp. 28-33, 2016.

[7] D. Zarifi, M. Soleimani, A. Abdolali, and S. E. Hosseininejad, "Parameter reconstruction of materials with off-diagonal anisotropy using the state transition matrix method," AEUInternational Journal of Electronics and Communications, Vol. 68, No. 9, pp. 877-882, 2014.

[8] S Daoudi, F Benabdelaziz, C Zebiri, D Sayad, FM Abdussalam, R. Abd-Alhameed. "Dispersion Characteristics of a Gyro-Chiro-Ferrite Shielded Multilayered Microstrip Line Using the Generalized Exponential Matrix," Internet Technologies and Applications (ITA), 12-15 Sept. 2017, pp $293-298$.

[9] K. Vytovtov, L. Mospan. Penetration effect in gyrotropic slab: Theory and applications. J. Opt. Soc. Am. A. Vol. 29, pp. 877882, 2012.

[10] E. Abdo-Sánchez, T. M. Martin-Guerrero \& C. CamachoPenalosa. Multiband Slot-Based Dual Composite Right/LeftHanded Transmission Line. Advanced Electromagnetics, Vol. 1(3), pp. 56-60, 2012.

[11] C. Zebiri, M. Lashab, and F. Benabdelaziz, "Effect of Anisotropic Magneto-Chirality on the Characteristics of a Microstrip Resonator," IET Microwaves, Antennas Propagation, Vol. 4, No. 4, pp. 446-452, 2010.

[12] A.M. Lerer, V.S. Mikhalevskii, and A.G. Shchuchinskii. Electrodynamic theory of a microstrip line on a ferrite substrate. Radiotekhnika i Elektronika. Vol. 29. No. 6, pp. 1039-1048, 1984.

[13] S. Aib, F. Benabdelaziz, C. Zebiri, and D. Sayad, "Propagation in Diagonal Anisotropic Chirowaveguides," Advances in OptoElectronics, Vol. 2017, 2017.

[14] F. Bayatpur, A.V Amirkhizi and S. Nemat-Nasser, "Experimental Characterization of Chiral Uniaxial Bianisotropic Composites at Microwave Frequencies," IEEE Transactions on Microwave Theory and Techniques, Vol.60, No. 4, pp. 1126-1135, 2012.

[15] C. Zebiri, S. Daoudi, F. Benabdelaziz, M. Lashab, et al. Gyrochirality effect of bianisotropic substrate on the operational of rectangular microstrip patch antenna. International Journal of Applied Electromagnetics and Mechanics. Vol. 51, pp. 249260, 2016.

[16] A. A. Sochava, C. R. Simovski, and S. A. Tretyakov. Chiral effects and eigenwaves in bi-anisotropic omega structures. Advances in complex electromagnetic materials. Springer, Dordrecht, 1997. 85-102, 1997. 
[17] S. Berthier. Anisotropic effective medium theories. J. Phys. I France 4 (1994) 303-318.

[18] M. Jaroszewski, S. Thomas, \& A. V. Rane. Advanced Materials for Electromagnetic Shielding: Fundamentals, Properties, and Applications. Wiley, 2018.

[19] U. C. Hasar, J. J. Barroso, C. Sabah, Y. Kaya, and M. Ertugrul, "Stepwise technique for accurate and unique retrieval of electromagnetic properties of bi-anisotropic metamaterials," J. Opt. Soc. Amer. B, Opt. Phys., vol. 30, pp. 1058-1068, Apr. 2013.

[20] U. C. Hasar, A. Muratoglu; M. Bute; J. J. Barroso; M. Ertugrul, "Effective Constitutive Parameters Retrieval Method for Bi-anisotropic Metamaterials Using Waveguide Measurements," IEEE Transactions on Microwave Theory and Techniques, vol. PP, no.99, pp.1-10, 2017.

[21] U. C. Hasar, G. Buldu, Y. Kaya and G. Ozturk, "Determination of Effective Constitutive Parameters of Inhomogeneous Metamaterials With Bi-anisotropy," in IEEE Transactions on Microwave Theory and Techniques, vol. 66, no. 8, pp. 3734-3744, Aug. 2018.

[22] T. Kitazawa, "Nonreciprocity of Phase Constants, Characteristic Impedances, and Conductor Losses in Planar Transmission Lines with Layered Anisotropic Media," IEEE Transactions on Microwave Theory and Techniques, Vol. 43, No. 2, pp. 445-451, 1995.

[23] Ž. J. Mančić, V. V. Petrovic. Strong FEM formulation for quasi-static analysis of shielded striplines in anisotropic homogeneous dielectric. Microwave and Optical Technology Letters. Vol. 54, pp. 1001-1006, 2012.

[24] A. N. A. Helal, K. Y. Elwasife, S. A. Taya. Characteristics of electromagnetic waves in slab waveguide structures comprising chiral nihility film and left-handed material claddings. Optik-International Journal for Light and Electron Optics. Vol. 149 332-343, 2017.

[25] H. Yang, Z. Y. Chen, and K. Y. Lv, "Analysis of Dispersion Characteristic of Microstrip Lines on Ferrite and Silicon Structures with Spectral-Domain Method," Applied Mechanics and Materials, Vol. 130, pp. 1244-1249. Trans Tech Publications, 2012.

[26] D. S. Mirshekar. Spectral domain method for microwave integrated circuits. John Wiley and Sons Inc; 1990.

[27] T. Itoh and R. Mittra, "A Technique for Computing Dispersion Characteristics of Shielded Microstrip Lines," IEEE Trans. Microwave Theory Tech., Vol. 22, pp. 896-898. Oct. 1974

[28] C. Zebiri, S. Daoudi, F. Benabdelaziz, M. Lashab, D. Sayad, \& R. A. Abd-Alhameed. Gyro-chirality effect of bianisotropic substrate on the resonant frequency and half-power bandwidth of rectangular microstrip patch antenna. CMC-Computers Materials \& Continua. Vol. 52, pp. 123-131, 2016.

[29] S. Daoudi, F. Benabdelaziz, C. Zebiri, and D. Sayad, "Generalized Exponential Matrix Technique Application for the Evaluation of the Dispersion Characteristics of a ChiroFerrite shielded Multilayered Microstrip Line," Progress In Electromagnetics Research M, Vol. 61, pp. 1-14, 2017.

[30] M. Aneesh, A. Kumar, A. Singh, and J. A. Ansari, Design and Analysis of Microstrip Line Feed Toppled T Shaped Microstrip Patch Antenna using Radial Basis Function Neural Network, " Journal of Electrical Engineering \& Technology, Vol. 10, No 2, pp. 634-640, 2015.

[31] D. Sayad, F. Benabdelaziz, C. Zebiri, S. Daoudi, and R. A. Abd-Alhameed, "Spectral Domain Analysis of Gyrotropic Anisotropy Chiral Effect on the Input Impedance of a Printed Dipole Antenna," Progress In Electromagnetics Research M, 51, pp. 1-8, 2016.
[32] W. C. Gibson. The method of moments in electromagnetics. NW: Chapman and Hall/CRC Taylor and Francis Group, 2008.

[33] R. F. Harrington.Field computation by moment methods. IEEE Press. 1993.

[34] J.C. Minor and D.M. Bolle. Modes in shielded microstrip on a ferrite substrate transversely magnetized in the plane of substrate. IEEE Transactions on Microwave Theory and Techniques, Vol. MTT-19. No. 7, pp. 570-577, 1971.

[35] P. L. Uslenghi. TE-TM decoupling for guided propagation in bianisotropic media. IEEE Trans. Antennas Propag. Vol. pp. 45, 284-286, 1997.

[36] T. Q. Ho, B. Beker. Spectral-domain analysis of shielded microstrip lines on biaxially anisotropic substrates. IEEE Transactions on Microwave Theory and Techniques. Vol. 39, pp. 1017-1021, 1991.

[37] M. K. Krage, G. I. Haddad. Frequency dependent characteristics of microstrip transmission lines. IEEE Trans. on Microwave Theory and Tech. Vol. 20, pp. 678-688, 1972.

[38] T. Itoh. Numerical techniques for microwave and millimeter wave passive structures. John Wiley and Sons. 1988. 